\title{
THE CONTINUITY OF FUNCTIONS OF MANY VARIABLES
}

\author{
BY \\ RICHARD KERSHNER
}

1. Introduction. It is known that a function $f(x, y)$ of two real variables may be continuous with respect to each variable separately throughout a given region without being continuous with respect to $(x, y)$ at all points of the region. In fact, W. H. and G. C. Young $\left.{ }^{1}\right)$ have given an example of a function $f(x, y)$ which is a continuous function of the position along every straight line in the unit square $[0,1] \times[0,1]$ but which has an uncountable number of two-dimensional discontinuities in every rectangle contained in the unit square. The example of W. H. and G. C. Young could easily be modified so as to yield a function continuous along every analytic arc but with an uncountable number of discontinuities in every rectangle.

If the number of variables is greater than two the situation becomes even worse. As was pointed out by Baire $\left({ }^{2}\right)$, for three variables, and subsequently by $\operatorname{Hahn}\left({ }^{3}\right)$, for any number of variables, a function $f\left(x_{1}, x_{2}, \cdots, x_{n}\right)$ may be continuous in each variable $x_{i}$ and yet be discontinuous with respect to $\left(x_{1}, x_{2}, \cdots, x_{n}\right)$ at every point of an $(n-2)$-dimensional rectangle. In fact let $g\left(x_{1}, x_{2}\right)$ be a function continuous in $x_{1}$ and $x_{2}$ separately but discontinuous at $(0,0)$. Then

$$
f\left(x_{1}, x_{2}, \cdots, x_{n}\right) \equiv g\left(x_{1}, x_{2}\right)
$$

is discontinuous at every point of the $(n-2)$-dimensional region $x_{1}=0, x_{2}=0$. Finally, Lebesgue $\left({ }^{4}\right)$ has shown that a function $f\left(x_{1}, x_{2}, \cdots, x_{n}\right)$ which is continuous in each variable $x_{i}$ separately may be of the $(n-1)$ st Baire class, although no worse.

The problem of considering how much could be said concerning the $n$-dimensional continuity points of a function $f\left(x_{1}, x_{2}, \cdots, x_{n}\right)$ which is assumed to be continuous in each $x_{i}$ separately was first treated in 1899 by Baire in the fundamental paper $\left(^{5}\right)$ in which he introduced most of the classic notions associated with his name. For the case of two variables his results were complete. He showed that

Presented to the Society, December 30, 1941; received by the editors April 16, 1942.

(1) W. H. Young and G. C. Young, Discontinuous functions continuous with respect to every straight line, Quart. J. Math. Oxford Ser. vol. 41 (1910) pp. 87-93.

(2) R. Baire, Sur les fonctions de variables réelles, Annali di Mathematica Pura ed Applicata vol. 3 (1899) pp. 1-122.

(3) Hahn, Über Funktionen mehrerer Veränderlichen, die nach jeder einzelnen Veränderlichen stetig sind, Math. Zeit. vol. 4 (1919) pp. 306-313.

(4) H. Lebesgue, Sur les fonctions représentable analytiquement, J. Math. Pure Appl. (6) vol. 1 (1905) pp. 139-212, cf. pp. 201, 202.

(5) Loc. cit. Footnote 2. 
(A) If $f(x, y)$ is, in the unit square, a continuous function of $x$, for fixed $y$, and a continuous function of $y$, for fixed $x$, then there is a residual set of lines parallel to each axis consisting entirely of points where $f(x, y)$ is continuous with respect to $(x, y)$.

Here and throughout the paper a set contained in an interval is called a residual set if its complement in that interval is of first category.

It should be recalled that, in consequence of Baire's classic theorem, a residual set in an interval is uncountably dense in every subinterval. Thus the statement obtained from (A) by reading "dense" for "residual" is an immediate consequence of (A). Actually Baire explicitly displayed only this weaker consequence although he actually proved (A). It follows immediately from (A) that

(B) Under the assumptions of (A) every line parallel to an axis contains a dense set of points where $f(x, y)$ is continuous with respect to $(x, y)$.

Clearly (A) is much stronger than (B). For example, (A) does, but (B) does not, imply that

(C) Under the assumptions of (A) the set of points where $f(x, y)$ is discontinuous with respect to $(x, y)$ has dimension (Menger) at most zero.

Of course this beautiful theorem $(\mathrm{C})$ was not stated by Baire, since the general notion of dimension involved is of a later date.

Baire also treated the case of three variables in the same 1899 paper. Unfortunately he was not able to generalize the result (A) (which, as is shown in $\$ 6$, is definitive) but only the weaker consequence (B). Of course, in view of the fact that $f(x, y, z)$ may be of the second Baire class, it is rather surprising that even (B) may be extended to the case of three variables. However, Baire was able to show that every plane parallel to a coordinate plane must contain a dense set of continuity points for $f(x, y, z)$, assumed continuous in $x, y$ and $z$ separately.

The methods of Baire apparently yielded nothing for $f\left(x_{1}, x_{2}, \cdots, x_{n}\right)$ if $n>3$, and it was not until 1910 that $\mathrm{Hahn}\left({ }^{6}\right)$ showed that, in spite of the increasing Baire class, a generalization of (B) remained valid for any $n$. In fact, it was shown that any $(n-1)$-dimensional hyperplane obtained by fixing one coordinate must contain a dense set of continuity points of a function $f\left(x_{1}, x_{2}, \cdots, x_{n}\right)$ assumed continuous in each $x_{i}$.

The only other consideration of the problem of which I am aware is a paper of Bogel $\left({ }^{7}\right)$ who established the conclusion of (B) under somewhat weaker assumptions than those of Baire.

(6) Loc. cit. Footnote 3.

( ${ }^{7}$ K. Bogel, Über die Stetigkeit und die Schwankung von Funktionen zweier reeler Veranderlichen, Math. Ann. vol. 81 (1920) pp. 64-93. 
The main purpose of this paper is to show that not only (B) but also the definitive result (A) can be generalized to the case of an arbitrary number of variables. The precise result, stated here for the case of three variables, is the following:

If $f(x, y, z)$ is, in the unit cube, a continuous function of $x$, and of $y$, and of $z$ then there is a residual set of planes parallel to each coordinate plane, on each of which there is a residual set of lines parallel to each possible coordinate axis consisting entirely of points where $f(x, y, z)$ is continuous with respect to $(x, y, z)$.

In particular the result to be established in the case of an arbitrary number of variables is strong enough to establish the natural generalization of $(C)$, that is,

If $f\left(x_{1}, x_{2}, \cdots, x_{n}\right)$ is, in the unit cube, a continuous function of each $x_{i}$, then the set of points where $f\left(x_{1}, x_{2}, \cdots, x_{n}\right)$ is discontinuous with respect to $\left(x_{1}, x_{2}, \cdots, x_{n}\right)$ has dimension at most $(n-2)$.

It will be recalled that the set of discontinuities may have dimension $n-2$ even if $f\left(x_{1}, x_{2}, \cdots, x_{n}\right)$ is an algebraic function.

The extension of the Baire results to the case of more than two variables is based mainly on a set-theoretic lemma (Lemma 2) which is proved in $\$ 2$. Section 2 also contains a list of the notations and results of a set-theoretic nature that will be needed later.

Section 3 contains a similar list of notations and results of a functiontheoretic nature that will be used. These are surprisingly few in number and elementary in nature.

In $\$ 4$ a greatly simplified proof is given of the Baire result for a function of two variables. This is technically unnecessary since the induction proof to be given in $\$ 5$ for the case of any number of variables could be based on the completely trivial case $n=1$ rather than the Baire case $n=2$, but the Baire result does not seem to be so well known as it deserves to be and it seemed that a direct modern proof might be useful.

In $\$ 6$ it is shown that the results of $\$ 5$ are the best possible. It seems not to have been known even that the Baire result (A) was best possible.

It might be wondered whether the results of $\$ 5$ can be strengthened by requiring more smoothness, for example, the existence of partial derivatives or a 1-dimensional Lipschitz condition, parallel to the axes. This problem is treated in $\$ 7$, where it is shown that an assumption considerably weaker than a 1-dimensional Lipschitz condition of any order $\alpha>0$ is sufficient to ensure that the discontinuities of $f\left(x_{1}, x_{2}, \cdots, x_{n}\right)$ are nowhere dense. This result seems to be new even in the simplest case $n=2$, although Bogel $\left({ }^{8}\right)$ has a much weaker result in this direction in the case $n=2$.

Finally, in $\$ 8$, it is shown that the result of $\$ 7$ is definitive and in fact that no further restrictions on the set of discontinuities are imposed by requiring any degree of smoothness, short of analyticity, parallel to the axes.

(8) K. Bogel, Über partiell differenzierbare Funktionen, Math.Zeit.vol.25(1926) pp.490-498. 
It might be mentioned that the results of this paper could easily be extended to quite abstract product spaces but the author's interests do not lie in that direction.

2. Preliminaries. In this section will be listed certain notations and results, some of them classic, that will be used in this paper. Special attention is called to the set-theoretic Lemma 2. which seems to be rather powerful.

Set. The bracket notation $[a ; \cdots]$ (or $[a \in S ; \cdots]$ ), is used to denote the set of all those elements $a$ (of $S$ ) for which the specified restrictions "..." are satisfied.

Interval. All intervals are understood to be open unless otherwise specified. The closed unit real interval $[0,1]$ is denoted by $U$ or $U_{1}$. The closed unit $n$-interval $U \times U \times \cdots \times U$ is denoted by $U_{n}$. If $I=(a, b)$ is a subinterval of $U$ then $|I|$ denotes its length $b-a$.

$F_{\sigma}$. A set is called an $F_{\sigma}$-set if it is the sum of a countable number of closed sets.

Dimension. The recursive (Menger-Urysohn) definition of dimension will be used. The empty set has dimension -1 . A set $S$ has dimension at most $m$ if each neighborhood of every point of $S$ contains another neighborhood of that point whose boundary intersects $S$ in a set with dimension at most $m-1$.

Category. A subset of $U_{n}$ is called of the first category if it is the sum of a countable number of sets nowhere dense in $U_{n}$. All other subsets of $U_{n}$ are called of the second category.

Residual. A subset of an interval of $U_{n}$ is called residual in that interval if its complement in that interval is of the first category.

BAIRE'S THFOREM. The empty set is not residual, that is, an interval is of the second category.

Baire's theorem will be used mainly in conjunction with one of the following two lemmas of which the first is classic (and trivial) and the second seems to be new.

LEMMA 1. Let $\left\{S_{j}\right\}$ be a sequence of sets in $U_{n}$ such that $S_{j}$ is closed and $\sum S_{j}$ is of the second category in $U_{n}$. Then, for some integer $k, S_{k}$ contains a subinterval of $U_{n}$.

Proof. Since $\sum S_{j}$ is of the second category, not all $S_{j}$ are nowhere dense. Thus some $S_{k}$ is dense in a subinterval $I$ of $U_{n}$. But $S_{k}$ is closed, so $S_{k} \supset I$.

In order to facilitate the statement of the other desired lemma, two definitions are given next.

Linearly closed. A set $S \subset U_{n}$ will be called linearly closed if it intersects each line parallel to a coordinate axis of $U_{n}$ in a closed set. Thus $S$ is linearly closed if, for each fixed

$$
\left(x_{1}, x_{2}, \cdots, x_{i-1}, x_{i+1}, \cdots, x_{n}\right) \in U_{n-1}
$$


the set

$$
\left[x_{i} \in U ;\left(x_{1}, x_{2} ; \cdots, x_{i-1}, x_{i}, x_{i+1}, \cdots, x_{n}\right) \in S\right]
$$

is closed.

Clearly a closed set is linearly closed, but not conversely.

Property $\Delta$. This will be defined for sets in $U_{n}$ inductively with respect to $n$. A set $S \subset U_{1}$ has property $\Delta$ if it is of second category in $U_{1}$. A set $S \subset U_{n}$ has property $\Delta$ if there is a set $R \subset U_{n-1}$ such that

and such that if

$$
R \text { has property } \Delta \text { in } U_{n-1}
$$

$$
x_{n-1} \equiv\left(x_{1}, x_{2}, \cdots, x_{n-1}\right) \in R
$$

then the set

$$
\left[x_{n} \in U ;\left(x_{n-1}, x_{n}\right) \in S\right]
$$

is of second category in $U$.

In view of Baire's theorem it is clear that $U_{n}$ itself has property $\Delta$ and $R$ may be chosen as $U_{n-1}$. More generally any set which contains a subinterval of $U_{n}$ has property $\Delta$ in $U_{n}$.

LEMMA 2. Let $\left\{S_{j}\right\}$ be a sequence of sets in $U_{n}$ such that $S_{j}$ is linearly closed and $\sum S_{j}$ has property $\Delta$. Then, for some $k, S_{k}$ contains a subinterval of $U_{n}$.

Proof. The proof is by induction on $n$. If $n=1$, Lemma 2 follows from Lemma 1. Suppose then that the case $n-1$ of Lemma 2 is valid.

Since $\sum S_{j}$ has property $\Delta$ there is a set $R \subset U_{n-1}$ such that

$$
R \text { has property } \Delta \text { in } U_{n-1}
$$

and

(2) $\quad x_{n-1} \in R$ implies $T \equiv\left[x_{n} ;\left(x_{n-1}, x_{n}\right) \in \sum S_{i}\right]$ of second category.

For fixed $x_{n-1} \in R$ let

$$
T_{j} \equiv T_{j}\left(x_{n-1}\right) \equiv\left[x_{n} ;\left(x_{n-1}, x_{n}\right) \in S_{j}\right], \quad j=1,2, \cdots .
$$

Then $T_{j}$ is closed since $S_{j}$ is linearly closed. Also $\sum T_{j}=T$ is of second category by (3), (2). Thus, by Lemma 1 , there is, for each $x_{n-1} \in R$, at least one integer $k$ and a corresponding interval $I \subset U$ such that $T_{k} \supset I$. Thus, if for each $x_{n-1} \in R$ and for each $k=1,2, \cdots$, one defines

$$
\lambda\left(\mathrm{x}_{n-1}, k\right) \equiv \max \left[|I| ; I \subset T_{k}\left(\mathrm{x}_{n-1}\right) \subset U\right]
$$

(which exists since $T_{k}$ is closed), then

$$
\text { for every } x_{n-1} \in R \text { there is a } k \text { such that } \lambda\left(x_{n-1}, k\right)>0 \text {. }
$$


Now, for each $j=1,2, \cdots ; k=1,2, \cdots$, let

$$
M_{j, k} \equiv\left[\mathrm{x}_{n-1} \in R ; \lambda\left(\mathrm{x}_{n-1}, k\right) \geqq 1 / j\right] .
$$

Then, clearly, $x_{n-1} \in M_{j, k}$ if and only if there is an interval $I=I\left(x_{n-1}, k\right) \subset U$, such that

$$
\left|I\left(x_{n-1}, k\right)\right| \geqq 1 / j \text { and } x_{n} \in I\left(x_{n-1}, k\right) \text { implies }\left(x_{n-1}, x_{n}\right) \in S_{k} .
$$

It will now be shown that the induction assumption may be applied to the sequence of sets $M_{j, k} \subset R \subset U_{n-1}$. First $\sum M_{j, k}=R$. In fact if $\mathrm{x}_{n-1} \in R$ then by (5), $\lambda\left(x_{n-1}, k\right) \geqq 1 / j$ for some $j, k$, and so $x_{n-1} \in \sum M_{j, k}$. Thus $\sum M_{j, k}$ has property $\Delta$ by (1).

It remains to show that $M_{j, k}$ is linearly closed. To this end it is sufficient to prove that if $\left\{x_{i, h}\right\}$ is, for fixed $1 \leqq i \leqq n-1$, a sequence of numbers in $U$ such that

$$
\lim _{h \rightarrow \infty} x_{i, h}=\bar{x}_{i} \text { exists }
$$

and

$$
\left(x_{1}, x_{2}, \cdots, x_{i-1}, x_{i, h}, x_{i+1}, \cdots, x_{n-1}\right) \in M_{j, k}, \quad h=1,2, \cdots,
$$

then

$$
\left(x_{1}, x_{2}, \cdots, x_{i-1}, \bar{x}_{i}, x_{i+1}, \cdots, x_{n-1}\right) \in M_{j, k} .
$$

It has been seen, in (6), that (8) implies the existence of an interval $I=I_{h} \subset U$ such that $\left|I_{h}\right| \geqq 1 / j$ and

$$
x_{n} \in I_{h} \text { implies }\left(x_{1}, x_{2}, \cdots, x_{i-1}, x_{i, h}, x_{i+1}, \cdots, x_{n-1}, x_{n}\right) \in S_{k} .
$$

Let $x_{n, h}$ denote the midpoint of $I_{h}$. Then there is a subsequence $x_{n, h_{m}}$ which is convergent, say

$$
x_{n, h_{m}} \rightarrow \bar{x}_{n} \text {. }
$$

Now let $x_{n}$ be a fixed number in $U$ such that

$$
\left|x_{n}-\bar{x}_{n}\right|<1 / 2 i
$$

so that

$$
\left|x_{n}-\bar{x}_{n}\right|=(1 / 2 j)-\delta
$$

for some $\delta>0$. Then, for sufficiently large $m$,

so that

$$
\left|x_{n, h_{m}}-\bar{x}_{n}\right|<\delta
$$

$$
\left|x_{n}-x_{n, h_{m}}\right|<1 / 2 j \text {. }
$$

Thus $x_{n} \in I_{h_{m}}$ for sufficiently large $m$. Then

$$
\left(x_{1}, x_{2}, \cdots, x_{i-1}, x_{i, h_{m}}, x_{i+1}, \cdots, x_{n-1}, x_{n}\right) \in S_{k},
$$


for sufficiently large $m$. Then, since $S_{k}$ is linearly closed,

$$
\left(x_{1}, x_{2}, \cdots, x_{i-1}, \bar{x}_{i}, x_{i+1}, \cdots, x_{n-1}, x_{n}\right) \in S_{k}
$$

by (7). But this is true for any fixed $x_{n}$ in the interval (9) of length $1 / j$. Thus

$$
\left(x_{1}, x_{2}, \cdots, x_{i-1}, \bar{x}_{i}, x_{i+1}, \cdots, x_{n-1}\right) \in M_{i, k}
$$

and it has been shown that $M_{j, k}$ is linearly closed.

The induction assumption may now be applied to the sequence $\left\{M_{j, k}\right\}$ and it is found that, for some integers $j_{1}, k_{1}$ the set $M_{j_{1}, k_{1}}$ contains a subinterval of $U_{n-1}$. Let $J_{1}$ be a closed subinterval of $U_{n-1}$ contained in $M_{j_{1}, k_{1}}$. Thus, for every point $x_{n-1}$ of the interval $J_{1}$ there is an interval $I=I\left(x_{n-1}\right) \subset U$ such that

$$
\left|I\left(x_{n-1}\right)\right| \geqq 1 / j_{1}
$$

and

$$
x_{n} \in I\left(x_{n-1}\right) \text { implies }\left(x_{n-1}, x_{n}\right) \in S_{k_{1}} .
$$

Now let $K_{j}$, for $j=1,2, \cdots, 2 j_{1}$, denote the interval

$$
K_{j}=\left((j-1) /\left(2 j_{1}\right), j /\left(2 j_{1}\right)\right) \subset U
$$

of length $1 /\left(2 j_{1}\right)$. Then let $N_{j}$, for $j=1,2, \cdots, 2 j_{1}$ be the set

$$
N_{j} \equiv\left[\mathrm{x}_{n-1} \in J_{1} ; x_{n} \in K_{j} \text { implies }\left(\mathrm{x}_{n-1}, x_{n}\right) \in S_{k_{1}}\right] \text {. }
$$

Then it is very easily seen that $N_{j}$ is linearly closed. On the other hand, if $x_{n-1} \in J_{1}$ then $I\left(x_{n-1}\right)$, being an interval of length at least $1 / j_{1}$ must contain some one of the intervals $K_{j}$. Then $x_{n-1} \in N_{j}$ for some $j$, that is, $\mathbf{x}_{n-1} \in \sum N_{j}$. Thus $\sum N_{j} \supset J_{1}$ and so $\sum N_{j}$ has property $\Delta$ by Baire's theorem. Thus, again applying the induction hypothesis, some $N_{j_{2}}$ contains a subinterval $J_{2} \subset J_{1} \subset U_{n-1}$. Then the subinterval $J_{2} \times K_{j_{2}}$ of $U_{n}$ is contained in $S_{k}$. This completes the proof of Lemma 2.

Another property of subsets of $U_{n}$ which is connected with property $\Delta$ will be useful later and is defined now.

Property $\Gamma$. Again the definition is inductive for sets in $U_{n}$. A set $S \subset U_{1}$ has property $\Gamma$ if it is residual in $U_{1}$. A set $S \subset U_{n}$ has property $\Gamma$ if there is a set $R \subset U_{n-1}$ such that

$$
R \text { has property } \Gamma \text { in } U_{n-1}
$$

and such that if $x_{n-1} \in R$ then the set

is residual in $U$.

$$
\left[x_{n} \in U ;\left(x_{n-1}, x_{n}\right) \in S\right]
$$

Clearly a set with property $\Gamma$ has also property $\Delta$, not conversely. However, there is a more striking connection expressed by the following: 
LemMa 3. $A$ set $S \subset U_{n}$ has property $\Gamma$ if and only if $U_{n}-S$ does not have property $\Delta$.

Proof. This can be proved by a straightforward induction. (If $n=1$ the statement reduces to the definition of a residual set.) The details of the proof suggest themselves readily.

Projection. If $S \subset U_{n}$ then, for each $i=1,2, \cdots, n$, the set of all

$$
\left(x_{1}, x_{2}, \cdots, x_{i-1}, x_{i+1}, \cdots, x_{n}\right) \in U_{n-1}
$$

such that there exists an $x_{i} \in U$ for which

$$
\left(x_{1}, x_{2}, \cdots, x_{i-1}, x_{i}, x_{i+1}, \cdots, x_{n}\right) \in S
$$

is called the projection of $S$ on $U_{n-1}^{\prime}$.

The last set-theoretic lemma that will be needed involves a connection between the property $\Delta$ and the dimension of a subset of $U_{n}$.

Lemma 4. Let $S \subset U_{n}$. Suppose that the projection of $S$ on $U_{n-1}^{i}$ for each $i=1,2, \cdots, n$, fails to have property $\Delta$. Then $S$ has dimension at most $n-2$.

Proof. Again this can be shown by a straightforward induction which it does not seem necessary to present in detail. It should be mentioned that one uses Lemma 3 and two well known facts about residual sets, namely that the product of two residual sets is residual and that a residual set is dense.

3. Further preliminaries. Throughout this section let $f\left(x_{1}, x_{2}, \cdots, x_{n}\right)$ $=f\left(x_{n-1}, x_{n}\right)=f\left(x_{n}\right)$ be defined in $U_{n}$ and be a continuous function of each $x_{i}$ for fixed $x_{1}, x_{2}, \cdots, x_{i-1}, x_{i+1}, \cdots, x_{n}$. In particular $f\left(x_{n-1}, x_{n}\right)$ is a continuous function of $x_{n}$ in $U$.

Unicontinuous. For convenience a function $f$ satisfying the above conditions will be referred to as unicontinuous.

$\delta_{\epsilon}\left(x_{n-1}\right)$. For fixed $x_{n-1} \in U_{n-1}, f$ is a uniformly continuous function of $x_{n}$, that is, for every $\epsilon>0$ there is a $\delta_{\epsilon}$ such that

$$
\left|x_{n}-\bar{x}_{n}\right| \leqq \delta_{\epsilon}
$$

implies

$$
\left|f\left(x_{n-1}, x_{n}\right)-f\left(x_{n-1}, \bar{x}_{n}\right)\right| \leqq \epsilon .
$$

It is apparent that for each $\epsilon>0$ and for fixed $x_{n-1}$, there is a greatest such $\delta_{\epsilon}$. This greatest $\delta_{\epsilon}$ will be denoted by $\delta_{\epsilon}\left(x_{n-1}\right)$.

Lemma 5. For each $\epsilon>0, \eta>0$ the set of points

$$
\left[x_{n-1} \in U_{n-1} ; \delta_{\epsilon}\left(x_{n-1}\right) \geqq \eta\right]
$$

is linearly closed.

Proof. This statement, which expresses the upper semi-continuity of 
$\delta_{\epsilon}\left(x_{1}, x_{2}, \cdots, x_{n-1}\right)$ with respect to each of $x_{1}, \cdots, x_{n-1}$, is reasonably well known and in any case is trivial.

$\Omega(f, S)$. If $S$ is a subset of $U_{n}$ then $\Omega(f, S)$ will denote

$$
\Omega(f, S) \equiv \text { l.u.b. }\left[f\left(\mathbf{x}_{n}\right) ; \mathbf{x}_{n} \in S\right]-\text { g.l.b. }\left[f\left(\mathbf{x}_{n}\right) ; \mathbf{x}_{n} \in S\right] .
$$

Clearly $S \subset T$ implies $0 \leqq \Omega(f, S) \leqq \Omega(f, T)$.

$\Omega\left(f, \mathbf{x}_{n}\right)$. If $\mathbf{x}_{n} \in U_{n}$ then $\Omega\left(f, \mathbf{x}_{n}\right)$ will denote

$$
\Omega\left(f, \mathrm{x}_{n}\right) \equiv \text { g.l.b. }\left[\Omega(f, S) ; S \text { is open and } \mathrm{x}_{n} \in S\right] \text {. }
$$

LeMma 6. If $\mathrm{x}_{n} \in U_{n}$ and $S_{n}$ is the open cube of side $1 / n$ centered at $\mathrm{x}_{n}$ then

$$
\Omega\left(f, \mathbf{x}_{n}\right)=\lim _{n \rightarrow \infty} \Omega\left(f, S_{n}\right) .
$$

LeмmA 7. The function $f$ is continuous at $\mathbf{x}_{n}$ if and only if $\Omega\left(f, \mathbf{x}_{n}\right)=0$.

LEMMA 8. For each $\eta>0$ the set

$$
\left[\mathbf{x}_{n} \in U ; \Omega\left(f, \mathbf{x}_{n}\right) \geqq \eta\right]
$$

is closed.

These three well known facts are stated only for reference.

4. Functions of two variables. This section will be devoted to the consideration of a unicontinuous function $f\left(x_{1}, x_{2}\right)$ defined in $U_{2}$. the set

THEOREM 1. Let $f\left(x_{1}, x_{2}\right)=f\left(x_{2}\right)$ be unicontinuous in $U_{2}$. Then for each $\eta>0$

$$
D_{\eta} \equiv\left[\mathrm{x}_{2} \in U_{2} ; \Omega\left(f, \mathrm{x}_{2}\right) \geqq \eta\right]
$$

has a nowhere dense projection on $U_{1}^{1}$ and $U_{1}^{2}$.

Proof. In view of the symmetry of the assumptions it is sufficient to prove that $D_{n}$ has a nowhere dense projection on $U_{1}^{1}$.

Let $\epsilon$ be fixed in $0<\epsilon<\eta / 4$. Then let

$$
S_{j} \equiv\left[x_{1} \in U_{1} ; \delta_{\epsilon}\left(x_{1}\right) \geqq 1 / j\right], \quad j=1,2, \cdots,
$$

where $\delta_{\epsilon}\left(x_{1}\right)=\delta_{\epsilon}\left(x_{2-1}\right)$ was defined in $\$ 2$. Then $S_{j}$ is closed, by Lemma 5 . Also $\sum S_{j}=U$ is of second category by Baire's theorem. Hence, by Lemma 1 , there is an integer $k$ and an interval $I \subset U$ such that $I \subset S_{k}$. Thus

$$
x_{1} \in I \text { implies } \delta_{\epsilon}\left(x_{1}\right) \geqq 1 / k \text {. }
$$

It will now be shown that if

$$
\left(x_{1}, x_{2}\right)=\mathrm{x}_{2} \in I \times U
$$

then

$$
\Omega\left(f, x_{2}\right) \leqq 4 \epsilon .
$$


To this end let $\bar{x}_{1} \in I$ and $\bar{x}_{2} \in U$. Then, since $f\left(x_{1}, \bar{x}_{2}\right)$ is a continuous function of $x_{1}$, there is a $\delta>0$ such that if $x_{1} \in\left(\bar{x}_{1}-\delta, \bar{x}_{1}+\delta\right)$ then

$$
\left|f\left(x_{1}, \bar{x}_{2}\right)-f\left(\bar{x}_{1}, \bar{x}_{2}\right)\right| \leqq \epsilon .
$$

Choose $\delta>0$ so small that $\left(\bar{x}_{1}-\delta, \bar{x}_{1}+\delta\right) \subset I$, as may be done since $I$ is open. Then, by (1), for each $x_{1} \in\left(\bar{x}_{1}-\delta, \bar{x}_{1}+\delta\right) \subset I$

$$
\left|f\left(x_{1}, x_{2}\right)-f\left(x_{1}, \bar{x}_{2}\right)\right| \leqq \epsilon
$$

provided

$$
\left|x_{2}-\bar{x}_{2}\right| \leqq 1 / k \leqq \delta_{\epsilon}\left(x_{1}\right) .
$$

Combination of the last two inequalities shows that the function value at any point $\left(x_{1}, x_{2}\right)$ in the rectangle

$$
J \equiv\left(\bar{x}_{1}-\delta, \bar{x}_{1}+\delta\right) \times\left(\bar{x}_{2}-1 / k, \bar{x}_{2}+1 / k\right)
$$

differs from the function value at $\left(x_{1}, x_{2}\right)$ by at most $2 \epsilon$. Thus

$$
\Omega(f, J) \leqq 4 \epsilon .
$$

(I do not pause over the modifications necessary at the boundary of $U_{2}$.) Thus, a fortiori,

$$
\Omega\left(f,\left(\bar{x}_{1}, \bar{x}_{2}\right)\right) \leqq 4 \epsilon<\eta .
$$

But $\left(\bar{x}_{1}, \bar{x}_{2}\right)$ was any point $I \times U$.

It has been shown that $U_{2}$ contains a strip $I \times U$ which contains no point of $D_{\eta}$. But the argument given was equally applicable to any substrip of $U_{2}$. This completes the proof of Theorem 1.

TheOREM 2. Let $f\left(x_{1}, x_{2}\right)$ be unicontinuous in $U_{2}$. Let $D$ denote the set of points in $U_{2}$ where $f\left(x_{1}, x_{2}\right)$ is discontinuous. Then $D$ is an $F_{\sigma}$-set and the projection of $D$ on $U_{1}^{1}\left(U_{1}^{2}\right)$ is of first category.

Proof. If $D_{1 / n}$ is the set

$$
D_{1 / n} \equiv\left[\mathrm{x}_{2} \in U_{2} ; \Omega\left(f, \mathrm{x}_{2}\right) \geqq 1 / n\right]
$$

then $D_{1 / n}$ is closed, by Lemma 8 . Also $D_{1 / n}$ has a nowhere dense projection on $U_{1}^{1}$ by Theorem 1 . Since $\sum D_{1 / n}=D$, by Lemma 7 , the proof is complete.

The next statement is the one which was called (A) in the introduction. It is clearly a restatement of Theorem 2.

THEOREM 3. Let $f\left(x_{1}, x_{2}\right)$ be unicontinuous in $U_{2}$. Then there is a residual set of lines parallel to each axis consisting entirely of points where $f\left(x_{1}, x_{2}\right)$ is continuous.

THEOREM 4. The set of discontinuities of a unicontinuous function in $U_{2}$ has dimension at most zero. 
Proof. This is immediate from Theorem 3 in view of the fact that a residual set is dense. Of course it follows from Lemma 4 also.

5. Functions of many variables. This section will be devoted to a proof of the appropriate generalization of the results of the last section for a unicontinuous function on $U_{n}$.

TheOREM 5. Let $f\left(x_{1}, \cdots, x_{n}\right)=f\left(x_{n}\right)$ be unicontinuous in $U_{n}$. Then for each $\eta>0$ the set

$$
D_{\eta} \equiv\left[\mathrm{x}_{n} \in U_{n} ; \Omega\left(f, \mathrm{x}_{n}\right) \geqq \eta\right]
$$

has a nowhere dense projection on $U_{n-1}^{i}$ for each $i=1,2, \cdots, n$.

Proof. Clearly it is sufficient to prove that the projection of $D_{\eta}$ on $U_{n-1}^{n}$ is nowhere dense.

Let $\epsilon$ be fixed in $0<\epsilon<\eta / 8$. Then let

$$
S_{j} \equiv\left[\mathrm{x}_{n-1} \in U_{n-1} ; \delta_{\epsilon}\left(\mathrm{x}_{n-1}\right) \geqq 1 / j\right], \quad j=1,2, \cdots .
$$

Then $S_{j}$ is linearly closed by Lemma 5 . Also $\sum S_{j}=U_{n-1}$ has property $\Delta$ in $U_{n-1}$ by Baire's theorem. Hence, by Lemma 2 , there is an integer $k$ and an interval $I_{n-1} \subset U_{n-1}$ such that $I_{n-1} \subset S_{k}$. Thus

$$
\mathbf{x}_{n-1} \in I_{n-1} \text { implies } \delta_{\epsilon}\left(x_{n-1}\right) \geqq 1 / k \text {. }
$$

Now suppose the theorem true for functions unicontinuous in $U_{n-1}$. For each fixed $x_{n} \in U$ let $f_{x_{n}}\left(x_{n-1}\right)$ denote the function $f\left(x_{n-1}, x_{n}\right)$ of $\mathbf{x}_{n-1}$. Let $\left\{x_{n, h}\right\}$ be a dense sequence in $U$. Finally let

$$
D_{\epsilon}^{h} \equiv\left[\mathbf{x}_{n-1} \in U_{n-1} ; \Omega\left(f_{x_{n, h}}, \mathbf{x}_{n-1}\right) \geqq \epsilon\right] .
$$

Then by the induction assumption $D_{\epsilon}^{h}$ has a nowhere dense projection on $U_{n-2}$ and, a fortiori, $D_{e}^{h}$ is nowhere dense in $U_{n-1}$. Thus, by Baire's theorem,

$$
\sum_{h} D_{\epsilon}^{h} \text { does not contain } I_{n-1} \text {. }
$$

Thus there is a point $\overline{\mathbf{x}}_{n-1} \in I_{n-1}$ such that $\overline{\mathbf{x}}_{n-1}$ is not in any $D_{e}^{h}$, that is, so that

$$
\Omega\left(f_{x_{n}, h}, \bar{x}_{n-1}\right)<\epsilon .
$$

It will now be shown that for every $x_{n} \in U$,

$$
\Omega\left(f,\left(\bar{x}_{n-1}, x_{n}\right)\right)<8 \epsilon .
$$

In fact let $\bar{x}_{n} \in U$ be fixed. Since $\left\{x_{n, h}\right\}$ is a dense sequence there is some $h_{1}$ such that

$$
\left|\bar{x}_{n}-x_{n, h_{1}}\right| \leqq 1 / 2 k \text {. }
$$

But, since $\overline{\mathbf{x}}_{n-1} \in I_{n-1}$, this means, by (1), 


$$
\left|\bar{x}_{n}-x_{n, n_{1}}\right| \leqq \delta_{\epsilon}\left(\bar{x}_{n-1}\right)
$$

so that

$$
\left|f\left(\bar{x}_{n-1}, \bar{x}_{n}\right)-f\left(\bar{x}_{n-1}, x_{n, h_{1}}\right)\right| \leqq \epsilon,
$$

by the definition of $\delta_{\varepsilon}\left(x_{n-1}\right)$. Now, by (2) and the definition of $\Omega\left(f_{x_{n, n}}, \bar{x}_{n-1}\right)$, there is an open neighborhood $J_{n-1}^{n_{1}}$ of $\bar{x}_{n-1}$ in $U_{n-1}$ such that

$$
\Omega\left(f_{x_{n, h_{1}}}, J_{n-1}^{h_{1}}\right)<2 \epsilon .
$$

Thus

(4) $\quad \overline{\bar{x}}_{n-1} \in J_{n-1}^{h_{1}}$ implies $\left|f\left(\overline{\mathbf{x}}_{n-1}, x_{n, h_{1}}\right)-f\left(\overline{\bar{x}}_{n-1}, x_{n, h_{1}}\right)\right|<2 \epsilon$.

Now suppose $J_{n-1}^{h_{1}}$ has been chosen so "small" that $J_{n-1}^{h_{1}} \subset I_{n-1}$ which is clearly permissible. Then

$$
\overline{\overline{\mathbf{x}}}_{n-1} \in J_{n-1}^{h_{1}} \subset I_{n-1} \text { implies } \delta_{\epsilon}\left(\overline{\bar{x}}_{n-1}\right) \geqq 1 / k \text {. }
$$

In other words

$$
\left|\overline{\bar{x}}_{n}-x_{n, h_{1}}\right| \leqq 1 / k \text { implies }\left|f\left(\overline{\bar{x}}_{n-1}, x_{n, h_{1}}\right)-f\left(\overline{\bar{x}}_{n-1}, \overline{\bar{x}}_{n}\right)\right| \leqq \epsilon .
$$

Combination of (3), (4), (5) gives

$$
\left|f\left(\overline{\mathbf{x}}_{n-1}, \bar{x}_{n}\right)-f\left(\overline{\bar{x}}_{n-1}, \overline{\bar{x}}_{n}\right)\right|<4 \epsilon
$$

whenever

$$
\overline{\bar{x}}_{n-1} \in J_{n-1}^{n_{1}} \text { and }\left|\overline{\bar{x}}_{n}-x_{n, h_{1}}\right| \leqq 1 / k
$$

where $h_{1}$ was determined so that

$$
\left|x_{n, h_{1}}-\bar{x}_{n}\right| \leqq 1 / 2 k \text {. }
$$

In particular if $\left|\overline{\bar{x}}_{n}-\bar{x}_{n}\right| \leqq 1 / 2 k$ the second requirement (6) is automatic. Thus for any point $\left(x_{n-1}, \overline{\bar{x}}_{n}\right)$ of the neighborhood

$$
I_{n} \equiv J_{n-1}^{h_{1}} \times\left(\bar{x}_{n}-1 / 2 k, \bar{x}_{n}+1 / 2 k\right)
$$

of $\left(\bar{x}_{n-1}, \bar{x}_{n}\right)$ the inequality $(6)$ is valid, Then,

$$
\Omega\left(f, I_{n}\right)<8 \epsilon
$$

and, a fortiori,

$$
\Omega\left(f, \bar{x}_{n}\right)<8 \epsilon .
$$

It has now been shown that the set

$$
\left[x_{n} \in U_{n} ; \Omega\left(f, x_{n}\right)<8 \epsilon\right]
$$

contains the line $\bar{x}_{n-1} \times U$. But (8) is an open set (cf. Lemma 8) in $U_{n}$ and so (8) contains a strip $J_{n-1} \times U$ for some interval $J_{n-1} \subset U_{n-1}$. 
The above argument gives the existence of a strip $J_{n-1} \times U$ in the complement of $D_{\eta} \subset D_{8 e}$ (since $\left.8 \epsilon<\eta\right)$. But this argument was equally applicable to any substrip of $U_{n}$. This completes the proof of Theorem 5 .

Exactly as in the case of two variables the following is an immediate consequence.

THEOREM 6. Let $f\left(x_{n}\right)$ be unicontinuous in $U_{n}$. Let $D$ denote the set of points in $U_{n}$ where $f\left(x_{n}\right)$ is discontinuous. Then $D$ is an $F_{\sigma}$-set and the projection of $D$ on $U_{n-1}^{i}$ is (for each $i=1,2, \cdots, n$ ) of first category.

This result, which is the natural extension of Theorem 2, does not obviously imply the desired generalization of Theorem 4. However, in view of Lemma 2, Theorem 6 is equivalent to the following:

THEOREM 7. Let $f\left(x_{n}\right)$ be unicontinuous in $U_{n}$. Let $D$ denote the set of points in $U_{n}$ where $f\left(x_{n}\right)$ is discontinuous. Then the projection of $D$ on $U_{n-1}^{d}$ (for each $i=1,2, \cdots, n)$ does not have property $\Delta$.

Proof. In view of Lemma 1 , an $F_{\sigma}$-set is of second category if and only if it contains an interval. In view of Lemma 2 and the fact that a closed set is linearly closed, an $F_{\sigma}$-set has property $\Delta$ if and only if it contains an interval. Thus, for $F_{\sigma}$-sets, first category is equivalent to the negation of property $\Delta$. This shows the equivalence of Theorems 6 and 7.

Of course Theorem 7 may be stated in a positive fashion similar to Theorem 3. It is this statement which was displayed, for the case $n=3$, in the introduction. Finally

THEOREM 8. The set of discontinuities of a unicontinuous function in $U_{n}$ has dimension at most $n-2$.

Proof. This is immediate from Theorem 7 and Lemma 4.

6. An example. In this section it will be shown that Theorem 6 describes the possible sets $D$ completely. This is done by proving the following.

THEOREM 9. Let $D$ be any $F_{a}$-set in $U_{n}$ such that the projection of $D$ on $U_{n-1}^{i}$ (for each $i=1,2, \cdots, n$ ) is of first category. Then there exists a unicontinuous function on $U_{n}$ for which $D$ is the set of discontinuity points.

The example which proves Theorem 9 will be constructed with the help of certain auxiliary functions whose existence is demonstrated first.

LEMMA 9. Let $D$ be any closed set in $U_{n}$ such that the projection of $D$ on $U_{n-1}^{i}$ (for each $i=1,2, \cdots, n$ ) is nowhere dense. Then there exists a function $f=f_{D}$ on $U_{n}$ such that
(a) $0 \leqq f_{D} \leqq 1$;
(b) $f_{D}$ is unicontinuous on $U_{n}$;
(c) $\Omega\left(f_{D}, x_{n}\right)=0$, if $x_{n} \in U_{n}-D$;
(d) $\Omega\left(f_{D}, x_{n}\right)=1$, if $x_{n} \in D$. 
Proof. By an oriented closed cube $K$ in $U_{n}$ will be understood a closed $n$-cube with faces parallel to the coordinate hyperplanes. With each oriented cube $K \subset U_{n}$ let there be associated a function $g_{K}$ with the following properties:

(1) $g_{K}$ is defined and continuous on $K$;

(2) $g_{K}=0$ on the boundary of $K$;

(3) $0 \leqq g_{K} \leqq 1$;

(4) $g_{K}=1$ at the midpoint of $K$.

For example $g_{K}\left(\mathrm{x}_{n}\right)$ might be chosen proportional to the distance from $\mathrm{x}_{n}$ to the boundary of $K$.

Let a line, parallel to the $i$ th coordinate axis of $U_{n}(i=1,2, \cdots, n)$ be called an $i$-grid line if it contains a point of $D$. Let $D_{i}^{*}$ be the set of all points which lie on some $i$-grid line. Then $D_{i}^{*}$ is clearly a closed set since $D$ is closed. Also $D_{i}^{*}$ has the same projection on $U_{n-1}^{i}$ as $D$, hence $D_{i}^{*}$ is nowhere dense in $U_{n}$.

Now let $D^{*} \equiv \sum_{i=1}^{n} D_{i}^{*}$. Then $D^{*}$ is a nowhere dense closed set in $U_{n}$. Let $C^{*}=U_{n}-D^{*}$, so that $C^{*}$ is an open set dense in $U_{n}$. It is well known that any open set in $U_{n}$ is the sum of a countable number of nonoverlapping oriented closed cubes. Thus $C^{*}=\sum K_{i}$ where $K_{i}$ is an oriented closed cube in $U_{n}$ and $K_{i}$ and $K_{j}$ have at most boundary points in common.

Notice that every point of $D$ is a limit point of midpoints of the $K_{i}$. In fact $\sum K_{i}=C^{*}$ is dense in $U_{n}$ and so each point of $D$ is a limit point of points in some collection of $K_{i}$. But any finite collection of $K_{i}$ form a closed set disjoint from $D^{*}$ and, a fortiori, disjoint from $D$. Thus each point of $D$ is a limit point of points from distinct $K_{i}$. But the diameters of any infinite collection of $K_{i}$ obviously must approach zero since $\sum K_{i} \subset U_{n}$. Thus each point of $D$ is a limit point of midpoints of $K_{i}$.

Now let $\left\{P_{j}\right\}$ be a sequence of points in $D$ such that every point of $D$ is either a point $P_{j}$ or a limit point of points $P_{j}$. The existence of such a sequence is quite obvious. Let a subsequence $\left\{K_{m, j}\right\}$ of the cubes $K_{i}$ be chosen in such a way that the midpoint of $K_{m, j}$ is at a distance at most $1 / m$ from $P_{j}$. The fact that $\left\{K_{m, j}\right\}$ exists is clear from the preceding paragraph. It is clear that by proceeding inductively with respect to $m+j$ the $K_{m, j}$ might be chosen as all distinct, but this is not essential.

Finally let $f=f_{D}$ be defined in $U_{n}$ by

(5) $\quad f_{D}\left(\mathbf{x}_{n}\right) \equiv\left\{\begin{array}{cl}g_{K_{m}, j}\left(\mathbf{x}_{n}\right) & \text { if } \mathbf{x}_{n} \in K_{m, j} ; \quad j=1,2, \cdots ; m=j, j+1, \cdots, \\ 0 & \text { if } \mathbf{x}_{n} \in U_{n}-\sum_{m \geqq j} K_{m, j} .\end{array}\right.$

It is to be shown that this function $f_{D}$ satisfies the requirements (a)-(d) of Lemma 9.

First of all (a) is obvious from (5) and (3).

In order to prove (d) let $P$ be a point in $D$. Then either $P$ is a point $P_{j}$ or $P$ 
is a limit point of such points. In the first case $P=P_{j}$ then $P$ is a limit point of the midpoints of the cubes $K_{m, j}$ for $m=j, j+1, \cdots$. Thus $P$ is a limit point of points where $f_{D}=1$ (in view of (5), (4)). But also $P$ is a limit point of boundary points of $K_{m, j}$ where $f_{D}=0$. Thus $\Omega\left(f_{D}, P\right)=1$. In the other case, that $P$ is the limit of some sequence $\left\{P_{j_{i}}\right\}$, then $P$ is also the limit of the midpoints of $K_{j_{i}, j_{i}}$ and again $\Omega\left(f_{D}, P\right)=1$. This establishes (d).

To prove (c), let $P$ be a point where $\Omega\left(f_{D}, P\right)>0$. Then $P$ is not an interior point of any cube $K_{m, j}$ by (1). Thus $f_{D}(P)=0$ (and in fact $P$ is a limit point of points where $\left.f_{D}=0\right)$. But, since $\Omega\left(f_{D}, P\right)=\delta>0, P$ must also be a limit point of points where $f_{D}>\delta / 2$ and hence a limit point of points in some subsequence $\left\{K_{m_{i} j_{i}}\right\}$ of $\left\{K_{m, j}\right\}$. This subsequence cannot contain only a finite number of distinct cubes since, if it did $P$ would clearly be an interior point of some $K_{m, j}$. Thus $P$ is the limit point of midpoints of an infinite sequence $\left\{K_{m_{i}, j_{i}}\right\}$ of distinct cubes. But each such midpoint is at a distance at most $1 / m_{i}$ from $P_{j_{i}}$. Thus $P$ is a point $P_{j_{i}}$ or a limit point of such points and, in either case, $P \in D$, since $D$ is closed.

Finally, to prove (b), the value of $f_{D}$ along lines parallel to the axes must be considered. If such a line is a grid line then it is contained entirely in $D^{*}$ and $f_{D} \equiv 0$ by (5). If such a line is not a grid line it is contained entirely in $U_{n}-D$ so that $f_{D}$ is continuous at every point by (c) and, a fortiori, is continuous along the given line.

The proof of Lemma 9 is now complete and it is now very easy to prove Theorem 9.

Proof of Theorem 9. Let $D$ be any $F_{\sigma}$-set in $U_{n}$ having a first category projection on $U_{n-1}^{i}$. Then, by the definition of an $F_{\sigma}$-set, $D=\sum D_{i}$, where $D_{i}$ is closed. But $D_{i}$ has a nowhere dense projection on each $U_{n-1}^{i}$. (In fact if a closed set is dense in some interval it contains the interval and so is not of first category by Baire's theorem.) Thus each $D_{\boldsymbol{i}}$ satisfies the requirement of the set $D$ in Lemma 9. Let $f_{D_{i}}$ be a function associated with $D_{i}$ satisfying (a)-(d) of Lemma 9. Then let

$$
f=\sum_{i=1}^{\infty} f_{D_{1}} / 3^{i}
$$

Then the series defining $f$ is uniformly convergent by (a). Thus $f$ is unicontinuous since all $f_{D_{i}}$ are unicontinuous. Also $f$ is continuous on $U_{n}-D=U_{n}-\sum D_{i}$ since each $f_{D_{j}}$ is continuous on $U_{n}-D_{j} \supset U_{n}-\sum D_{i}$. Finally, at any point $P$ of $D, f$ is discontinuous since the convergence factors $1 / 3^{i}$ were chosen suffciently rapid that "cancellation" of the discontinuities is impossible in view of $(\mathrm{d})$.

7. The consequence of stronger assumptions. This section and the following one are devoted to an investigation of the consequence of assuming stronger smoothness than simply continuity parallel to the axes. In order 
to state the precise requirement that will be considered in this section, a definition is needed first.

Let $f\left(x_{n}\right)$ be a unicontinuous function in $U_{n}$, so that $f\left(x_{n-1}, x_{n}\right)$ is a uniformly continuous function of $x_{n}$ in $U$. Let

$$
\omega\left(x_{n-1}, \delta\right)=\max _{\left|x_{n}-x_{n}^{\prime \prime}\right| \leqq \delta}\left|f\left(x_{n-1}, x_{n}^{\prime}\right)-f\left(x_{n-1}, x_{n}^{\prime \prime}\right)\right| \text {. }
$$

Then $\omega\left(x_{n-1}, \delta\right)$ is, for each fixed $x_{n-1}$, a monotone non-decreasing function of $\delta$ in $0<\delta \leqq 1$ with $\omega\left(x_{n-1}, 0+0\right)=0$.

Condition S: The function $f\left(x_{n-1}, x_{n}\right)$ of $x_{n}$ is said to satisfy condition $\mathrm{S}$ if there exists a sequence $\left\{\omega_{m}(\delta)\right\}$ of functions $\omega_{m}(\delta)$ defined for $0<\delta \leqq 1$ such that

$$
\omega_{m}(0+0)=0
$$

and, for every $x_{n-1} \in U_{n-1}$ there is an integer $m$ such that

$$
\omega\left(x_{n-1}, \delta\right) \leqq \omega_{m}(\delta),
$$$$
0<\delta \leqq 1
$$

For example if, for each fixed $x_{n-1}, f$ satisfies a Lipschitz condition of order one, such a sequence is provided by $\omega_{m}(\delta)=m \cdot \delta$. If it is known only that for each $\mathrm{x}_{n-1}, f$ satisfies a Lipschitz condition of some positive order then $\omega_{m}(\delta)=m \cdot \delta^{1 / m}$ is a sequence of the required type. By considering such sequences as $\omega_{m}(\delta)=1 /|\log \delta|^{1 / m}$ it is seen that condition $S$ is very weak. However the theorem to be proved next, taken in conjunction with the example of the preceding section, implicitly shows that condition $\mathrm{S}$ is not always satisfied.

THEOREM 10. Let $f\left(x_{n}\right)$ be a unicontinuous function on $U_{n}$ which satisfies condition $\mathrm{S}$ with respect to any one of its variables as $x_{n}$. Let $D$ denote the set of points in $U_{n}$ where $f\left(x_{n}\right)$ is discontinuous. Then $D$ is an $F_{\sigma}$-set and the projection of $D$ on $U_{n-1}^{i}$ is (for each $i=1,2, \cdots, n$ ) nowhere dense.

Proof. The proof is by induction based on the completely trivial case $n=1$. Suppose then that the theorem is true for the case $n-1$.

Now let

$$
S_{j}=\left[\mathbf{x}_{n-1} ; \omega\left(\mathbf{x}_{n-1}, \delta\right) \leqq \omega_{j}(\delta)\right] .
$$

Then $S_{j}$ is easily seen to be linearly closed. Also $\sum S_{j}=U_{n-1}$ in view of condition $S$. Thus Lemma 2 can be applied to the sequence $\left\{S_{j}\right\}$ and one finds that there is an integer $k$ and a subinterval $I_{n-1} \subset U_{n-1}$ such that $I_{n-1} \subset S_{k} \subset U_{n-1}$.

Let $\bar{x}_{n} \in U$ be fixed. Then $f\left(x_{n-1}, \bar{x}_{n}\right)$, considered as a function of $\mathrm{x}_{n-1}$, has, by the induction hypothesis, discontinuities at a set of $x_{n-1}$ with a nowhere dense projection and, a fortiori, nowhere dense in $U_{n-1}$. Thus there is an interval $J_{n-1} \subset I_{n-1}$ where $f\left(x_{n-1}, \bar{x}_{n}\right)$ is a continuous function of $x_{n-1}$. Thus if $\overline{\mathbf{x}}_{n-1} \in J_{n-1}$ then for every $\epsilon>0$ there is a $\delta_{\epsilon}$ such that

$$
\begin{gathered}
\left|\bar{x}_{n-1}-x_{n-1}\right|<\delta_{\epsilon} \text { implies } x_{n-1} \in J_{n-1} \text { and } \\
\left|f\left(\bar{x}_{n-1}, \bar{x}_{n}\right)-f\left(x_{n-1}, \bar{x}_{n}\right)\right|<\epsilon / 2 .
\end{gathered}
$$


Now let $\bar{\delta}>0$ be chosen so that

$$
\omega_{k}(\bar{\delta}) \leqq \epsilon / 2
$$

which is possible since $\omega_{k}(0+0)=0$. Then, for any $x_{n-1} \in J_{n-1} \subset I_{n-1} \subset S_{k}$,

$$
\omega\left(x_{n-1}, \bar{\delta}\right) \leqq \omega_{k}(\bar{\delta}) \leqq \epsilon / 2 .
$$

Thus, by the definition of $\omega\left(x_{n-1}, \delta\right)$,

$$
\left|\bar{x}_{n}-x_{n}\right|<\bar{\delta} \text { implies }\left|f\left(\mathbf{x}_{n-1}, \bar{x}_{n}\right)-f\left(\mathbf{x}_{n-1}, x_{n}\right)\right| \leqq \epsilon / 2 \text {. }
$$

From (1) and (2), one has

$$
\left|f\left(\overline{\mathbf{x}}_{n-1}, \bar{x}_{n}\right)-f\left(\mathbf{x}_{n-1}, x_{n}\right)\right| \leqq \epsilon
$$

if $\left|\overline{\mathbf{x}}_{n-1}-x_{n-1}\right|<\delta_{\epsilon}$ and $\left|\bar{x}_{n}-x_{n}\right|<\bar{\delta}$. Hence $f$ is continuous at $\left(\bar{x}_{n-1}, \bar{x}_{n}\right)$. But $\left(\bar{x}_{n-1}, \bar{x}_{n}\right)$ was arbitrary in $J_{n-1} \times U$.

It has been shown that $U_{n}$ contains a strip $J_{n-1} \times U$ consisting entirely of continuity points of $f$. But the argument leading to the existence of this strip is equally applicable to any substrip of $U_{n}$. Thus the discontinuity points of $f$ have a nowhere dense projection on $U_{n-1}^{n}$. The symmetry of the assumptions shows that the proof is complete.

8. Another example. In this section it will be shown that the conclusion of Theorem 10 cannot be strengthened (as far as a restriction on $D$ is concerned) even if the assumptions are strengthened to the point of requiring the existence of all derivatives along any line parallel to an axis. The precise statement of the theorem to be established follows.

THEOREM 11. Let $D$ be any $F_{\sigma}$-set in $U_{n}$ such that the projection of $D$ on $U_{n-1}^{i}$ (for each $i=1,2, \cdots, n)$ is nowhere dense. Then there exists a function $f\left(x_{n}\right)$ on $U_{n}$ such that $f\left(x_{n}\right)$, considered as a function of any one of the variables (for fixed values of the remaining $n-1$ variables) has all derivatives and such that $D$ is the set of discontinuity points of $f\left(\mathrm{x}_{n}\right)$.

Proof. The construction of the example which proves Theorem 11 is similar to the construction in the proof of Lemma 9 and will not be given in quite so much detail.

First let $\bar{D}$ be the closure of $D$. Then $\bar{D}$ has a nowhere dense projection on each $U_{n-1}^{i}$. As in the proof of Lemma 9 let $\bar{D}_{i}^{*}$ be the set of points lying on some " $i$-grid line," that is, some line parallel to the $x_{i}$-axis containing a point of $\bar{D}$. As before, let $\bar{D}^{*}=\sum_{i=1}^{n} \bar{D}_{i}^{*}$. Finally let $\bar{C}^{*}=U_{n}-\bar{D}^{*}$, so that $\bar{C}^{*}$ is a dense open set in $U_{n}$. Now $\bar{C}^{*}=\sum K_{i}$ where $K_{i}$ is a closed oriented cube and $K_{i}$ and $K_{j}$ have at most boundary points in common.

With any closed oriented cube $K \subset U_{n}$ let there be associated a function $g_{K}$ which, in addition to satisfying the requirements (1)-(4) of $\$ 6$, is infinitely differentiable along any line parallel to an axis, with all derivatives vanishing at the boundary of $K$. (Of course, at the boundary of $K$ these derivatives are 
all one-sided derivatives calculated with respect to the interior of $K$.) For example, if $K$ is the unit cube $U_{n}$ then $g_{K}$ might be chosen as

$$
g_{K}\left(x_{1}, x_{2}, \cdots, x_{n}\right)=\exp (8 n) \prod_{i=1}^{n} \exp \left(-1 / x_{i}^{2}\right) \cdot \exp \left(-1 /\left(x_{i}-1\right)^{2}\right) .
$$

Now let the $F_{\sigma}$-set $D$ be written as $D=\sum D_{k}$ where $D_{k}$ is closed. For each $k$ let $\left\{P_{k, j}\right\}$ be a sequence of points in $D_{k}$ such that every point of $D_{k}$ is either a point $P_{k, j}$ or a limit point of such points. Also let $\left\{K_{m, k, j}\right\}$ be a subsequence of the cubes $K_{i}$ such that the midpoint of $K_{m, k, j}$ is at a distance at most $1 / m$ from $P_{k, j}$. This time it is important to choose the $K_{m, k, j}$ all distinct.

Now a function $f\left(x_{n}\right)$ satisfying the requirements of Theorem 11 can be defined by

$$
f\left(\mathbf{x}_{n}\right)=\left\{\begin{array}{cll}
(1 / k) g_{K_{m, k}, j}\left(\mathbf{x}_{n}\right) & \text { if } \mathbf{x}_{n} \in K_{m, k, j}, & m \geqq \max (k, j) ; \\
0 & \text { if } \mathbf{x}_{n} \in U_{n}-\sum_{m \geqq k, j} K_{m, k, j} .
\end{array}\right.
$$

The fact that this function is discontinuous on $D$ and continuous on $U_{n}-D$ is verified very much as it was shown that the function $f_{D}$ defined in the proof of Lemma 9 had $D$ as its set of discontinuities. In the present case $D_{K}$ turns out to be the set of points $P$ where $\Omega(f, P) \geqq 1 / k$. To see that $f$ is infinitely differentiable along any line parallel to a coordinate axis, notice that such a line lies either in $\bar{D}^{*}$ or in $\bar{C}^{*}$. In the first case $f \equiv 0$. In the second case the given line is at a finite distance $\delta>0$ from the closed set $\bar{D}^{*}$ and, a fortiori, at a distance at least $\delta>0$ from any point of $D \subset \bar{D}^{*}$. Thus at most a finite number of the cubes $K_{m, k, j}$, with $m \geqq \max (k, j)$, are intersected by the given line. Thus along the given line $f$ is zero save for a finite number of nonoverlapping intervals in which it is modified by inserting an infinitely differentiable piece with all derivatives vanishing at the end points. This completes the proof.

The Johns Hopkins University, BALTIMORE, MD. 